\title{
Gamma Knife Radiosurgery does not alter the copy number aberration profile in sporadic vestibular schwannoma
}

\author{
Aril Løge Håvik ${ }^{1,2,4}\left(\right.$ D $\cdot$ Ove Bruland ${ }^{2} \cdot$ Dhanushan Dhayalan $^{3,4} \cdot$ Morten Lund-Johansen $^{3,4} \cdot$ Per-Morten Knappskog ${ }^{1,2}$
}

Received: 28 May 2020 / Revised: 20 September 2020 / Accepted: 22 September 2020

(c) The Author(s) 2020, corrected publication 2021

\begin{abstract}
Introduction Ionizing radiation is a known etiologic factor in tumorigenesis and its role in inducing malignancy in the treatment of vestibular schwannoma has been debated. The purpose of this study was to identify a copy number aberration (CNA) profile or specific CNAs associated with radiation exposure which could either implicate an increased risk of malignancy or elucidate a mechanism of treatment resistance.

Methods 55 sporadic VS, including 18 treated with Gamma Knife Radiosurgery (GKRS), were subjected to DNA wholegenome microarray and/or whole-exome sequencing. CNAs were called and statistical tests were performed to identify any association with radiation exposure. Hierarchical clustering was used to identify CNA profiles associated with radiation exposure.

Results A median of 7 (0-58) CNAs were identified across the 55 VS. Chromosome 22 aberration was the only recurrent event. A median aberrant cell fraction of $0.59(0.25-0.94)$ was observed, indicating several genetic clones in VS. No CNA or CNA profile was associated with GKRS.

Conclusion GKRS is not associated with an increase in CNAs or alteration of the CNA profile in VS, lending support to its low risk. This also implies that there is no major issue with GKRS treatment failure being due to CNAs. In agreement with previous studies, chromosome 22 aberration is the only recurrent CNA. VS consist of several genetic clones, addressing the need for further studies on the composition of cells in this tumor.
\end{abstract}

Keywords Vestibular schwannoma $\cdot$ Gamma Knife Radiosurgery $\cdot$ Whole genome microarray $\cdot$ Intratumor genetic heterogeneity $\cdot$ Neurosurgery $\cdot$ Genetics

\section{Introduction}

Morten Lund-Johansen and Per-Morten Knappskog have contributed equally to this work.

Morten Lund-Johansen

mljo@helse-bergen.no

$\bowtie$ Per-Morten Knappskog per.knappskog@helse-bergen.no

1 Department of Clinical Science, University of Bergen, Bergen, Norway

2 Center for Medical Genetics and Molecular Medicine, Haukeland University Hospital, Bergen, Norway

3 Department of Clinical Medicine, University of Bergen, Bergen, Norway

4 Department of Neurosurgery, Haukeland University Hospital, Bergen, Norway
Vestibular schwannoma (VS) is a benign intracranial neoplasm originating from the Schwann cells surrounding the vestibular portion of the 8 th cranial nerve. VS makes up $8 \%$ of intracranial tumors, with an annual incidence rate ranging from 10 to 22 per million $[1,2]$. Although patients have a normal life expectancy, they experience significantly reduced quality of life attributable to dizziness, headache, hearing loss, facial nerve palsy and tinnitus [3]. Inactivation of the NF2 tumor suppressor gene is considered an initiating event in VS tumorigenesis, but it is likely that other factors also contribute $[4,5]$. During recent years, several novel genetic events have been linked to the disease [6-8].

Gamma Knife Radiosurgery (GKRS) is a type of ionizing radiation therapy commonly used to treat VS. There are controversies regarding whether ionizing radiation might induce malignant degeneration or second neoplasms $[9,10]$. 
The risk for inducing neoplasms following ionizing radiation demonstrates a linear dose-response relationship, thus making it theoretically feasible for GKRS inducing neoplasms in the normal tissue surrounding VS [11].

The aim of this study was to analyze the genome of 55 sporadic VSs (sVS) to assess whether GKRS induce copy number aberrations (CNA). To understand the mechanism, we wanted to evaluate whether there are any genetic aberrations associated with GKRS treatment resistance. Previous studies on structural changes in the VS genome have identified chromosome 22q loss as the only recurring event, present in $25-83 \%$ of VSs [12]. However, previous studies have used techniques with lower resolution. Hence, our secondary aim was to characterize CNAs at a more detailed level as well as using this data to evaluate intratumor heterogeneity.

\section{Materials and methods}

\section{Patient samples}

VS tissue and matched blood sample was collected from 55 patients without a history of NF2, who underwent first-time suboccipital resection of unilateral VS at the Department of Neurosurgery, Haukeland University Hospital, from August 2003 to May 2017. Eighteen patients had been previously treated with GKRS for the same VS. Written informed consent was received from all patients before tissue harvesting and the study was approved by the Regional Ethical Committee for medical research in Western Norway (2013/374). Tumor samples were harvested from the subcapsular part and snap frozen and stored in liquid nitrogen in the Bergen Neurosurgical Tissue Bank at Haukeland University Hospital. All samples underwent routine histology. Volumetric tumor measurements were performed on BrainLab Elements if preoperative MRI scans were available (Version 2.4.0, BrainLab AG, Munich, Germany).

\section{DNA extraction}

DNA was extracted by disrupting the tumor tissue with the TissueLyser (Qiagen, Hilden, Germany) followed by protease treatment. DNA was then extracted using the QIAamp DNA Mini Kit (Qiagen). The DNA quality and quantity were evaluated with $1 \%$ SeaKem gel electrophoresis and NanoDrop (Thermo Fisher Scientific), respectively.

\section{Whole-genome DNA microarray}

The CytoScan HD microarray (Affymetrix, UK) was used to detect chromosomal aberrations according to the manufacturer's recommendations. CNAs were called using three different software: (1) chromosome analysis suite v3.2
(ChAS, Affymetrix, UK), (2) Rawcopy [13] and (3) Nexus Copy Number (BioDiscovery, El Segundo, CA, USA). All data were mapped to the hg 19 reference genome build. We applied the following filtering criteria for including the called CNAs in downstream analysis: (1) marker count $\geq 90$ for gains; (2) marker count $\geq 30$ for losses; (3) visual confirmation for mosaic variants; (4) segment size $\geq 1 \mathrm{Mbp}$ for copy number neutral runs of homozygosity (CNN-ROH). Recurrent CNN-ROHs were further inspected for harboring small variants in whole-exome sequencing (WES) data using IGV[14]. BEDTools was used to produce a per sample union CNA call set, merge fragmented calls and to identify common regions harboring CNA across the cohort [15]. Candidate CNAs were manually inspected in IGV and filtered based on the following criteria: (1) variant not present in databases of copy number variants (CNV) in normal healthy controls (Affymetrix reference database with $\mathrm{n}=2691$, Database of genomic variants as per May 2016 [16]); (2) variant containing NCBI reference sequence gene; (3) variant present in 3 or more samples. GISTIC [17] was used to identify statistically significant aberrated regions across the cohort.

For estimating aberrant cell fraction and allele specific copy number profiles in the tumors, the Allele-Specific Copy number Analysis of Tumors 2.5.2 (ASCAT) software was used [18]. Per sample log ratio (LR) and B-allele frequency (BAF) values from the 27 tumors analyzed with Rawcopy was used for input. ASCAT was run with default parameters except from gamma which was set to 0.45 in compliance with the estimated compression factor in the Affymetrix CytoScan HD microarray.

\section{Whole-exome sequencing (WES)}

WES data were available from a previous study on 46 sVSs, including 18 samples also analyzed with microarray [7]. The Sequenza software version 2.1.2 was used for estimating aberrant cell fraction and calling allele-specific copy number profiles from the BAM files [19]. The 18 samples analyzed with both microarray and WES were used as training data to set the following parameters for running Sequenza: gamma $=100, \mathrm{kmin}=30$ and median normalization method. Aberrant cell fraction estimates below 0.20 were not included as the software was not trained to estimate at this level.

\section{Statistical analyses}

Statistical analyses, including descriptive statistics, contingency table statistics, Mann-Whitney $\mathrm{U}$ test and linear correlation, were done using Nexus Copy Number and/or $\mathrm{R}$ [20]. Clustering of the sample set based on CNA profiles was done with Rawcopy using the hclust $\mathrm{R}$ package as well 
as with the built-in complete linkage hierarchical clustering algorithm in Nexus Copy Number.

\section{Results}

\section{Patient characteristics}

55 patients presenting with sVS were included (Table 1). Mean age at the time of surgery was 53.3 years ranging from 18 to 80 years. Mean preoperative tumor volume was $8.4 \mathrm{~cm}^{3}$ ranging from 0.37 to $26.78 \mathrm{~cm}^{3} .18$ patients underwent Gamma Knife Radiosurgery (GKRS) prior to surgical removal of the primary tumor. Mean time between GKRS and surgery was 1429 days ranging from 280 to 3478 days, and the margin dose in all cases was $12 \mathrm{~Gy}$. All but three GKRS treated patients needed surgical removal because of post-treatment growth; VS14 experienced dizziness, VS16 acquired an intratumoral cyst and VS26 developed trigeminal neuralgia. Five patients had cystic tumors.

\section{Chromosome 22 aberration is the only recurrent copy number aberration in sVS}

Using the union call set from filtered ChAS and Rawcopy segments, a median of 7 (0-58) CNAs per sample was identified. Figure 1 illustrates the karyogram of a representative sVS. A median of $0.17 \%$ of the sVS autosome was affected by CNA. 38 genomic loci were found to harbor a CNA in three or more samples. However, all but the chromosome 22 loss were common variants $(\mathrm{CNV})$ present in healthy controls. A median of $3(0-134) \mathrm{CNN}-\mathrm{ROH}$ were seen in the tumors. None of the recurrent regions across the samples harbored any point mutations or indels. ASCAT was then used to infer aberrant cell fraction and absolute allele specific copy number. The number of CNAs identified by the different approaches were highly correlated $(r=0.831$, $\mathrm{p}<0.001)$. ASCAT identified a median of $21(2-219)$ autosomal CNAs with a median gain-to-loss ratio of 1.25 (0.25-6.50). The only recurrent CNA retained after filtering was chromosome 22 loss or CNN-ROH. GISTIC analyses on segmented data from Rawcopy and Nexus Copy Number identified chromosome 22 loss as a significantly recurrent event $\left(\mathrm{Q}\right.$-bound $=1.36 \times 10^{-9}, \mathrm{G}$-score $\left.=15.72\right)$. Other events identified were either CNVs or non-coding DNA. Neither tumor volume, volumetric growth nor age was significantly associated with chromosome 22 status, number of CNAs or aberrant cell fraction. Aggregating the results from ASCAT and Sequenza, 25 out of 55 (45\%) tumors harbored a chromosomal aberration at chromosome 22 including seven tumors with CNN-ROH, 17 tumors with loss and one tumor with a loss followed by a CNN-ROH (Table 2). Most aberrations encompassed all the analyzed probes on the chromosome suggesting a total loss of the chromosome. The chromosome 22 aberrated group was comparable to the entire cohort with regards to sex distribution, GKRS exposure, age, tumor volume and time elapsed from GKRS to surgery. Combining the structural variants identified in this study with whole-exome sequencing and multiplex ligationdependent amplification (MLPA) data from our previous study, 41 out of 55 (75\%) harbored at least one NF2 mutation [7]. When only including the samples that were analyzed with whole-exome sequencing, 38 out of 46 (83\%) harbored at least one mutation including 13 samples with one hit and 25 samples with 2 hits.

\section{Mosaic chromosome 22 loss reveals intratumor genetic heterogeneity in sVS}

Among the chromosome 22 aberrated tumors, a median aberrant cell fraction of $0.59(0.25-0.94)$ was observed (Table 2). Aberrant cell fraction did not correlate with tumor growth. The level of mosaicism is demonstrated in the splitting of the BAF signal (Fig. 2). A Chi-square test demonstrated that tumors with aberrated chromosome 22 were significantly more likely to be estimated as heterogeneous $\left(\chi^{2}=22.212\right.$, Fisher's $\left.p=0.000\right)$. Among the four tumors with diploid chromosome 22 and estimated aberrant cell fraction below 1, one tumor had several CNN-ROHs, one tumor harbored another large CNA [del [21] (q11.2q22.3)], whereas the two other tumors were estimated to have an aberrant cell fraction between 0.95 and 1 . It seems likely that ASCAT and Sequenza are dependent on a large CNA to estimate aberrant cell fraction and hence only estimates from chromosome 22 aberrant tumors were included for reporting (Table 2). In four chromosome 22 aberrated tumors, an aberrant cell fraction could not be estimated, and all these tumors had WES data only.

Among the 46 tumors analyzed with WES, a total of 45 small nucleotide variants and indels were found with a median variant allele frequency of $24 \%$ [7]. We found a positive correlation between the estimated aberrant cell fraction from the chromosome 22 aberrated tumors and variant allele frequency $\left(\operatorname{adj} R^{2}=0.43, p=0.006\right)$.

\section{GKRS does not alter the copy number profile of sVS}

A Chi-square test of independence demonstrated no difference in the frequency of chromosome 22 aberration in irradiated $(39 \%)$ and radiation-naïve $(49 \%)$ tumors $\left(\chi^{2}=0.155\right.$, Fisher's $\left.p=0.572\right)$. We found no differences in aberrant cell fraction, number of CNAs, type of CNA or the portion of the genome covered by CNAs between the irradiated and radiation-naïve tumors. The clustering algorithms applied demonstrated that clusters identified did not rely on previous radiation exposure (Fig. 3). Using 
Table 1 Patient demographics. Patient demographics of 55 vestibular schwannomas

\begin{tabular}{|c|c|c|c|c|c|c|}
\hline ID & GKRS $^{1}$ & Age & Volume $^{2}$ & Sex & Microarray $^{3}$ & WES $^{4}$ \\
\hline VS1 & na & 58 & 4.25 & $\mathrm{~F}$ & $X$ & $X$ \\
\hline VS2 & na & 61 & NA & M & $\mathrm{X}$ & $X$ \\
\hline VS3 & na & 68 & 16.34 & M & $X$ & $X$ \\
\hline VS4 & na & 67 & 3.17 & $\mathrm{~F}$ & $\mathrm{X}$ & $\mathrm{X}$ \\
\hline VS5 & na & 58 & 12.82 & M & $\mathrm{X}$ & $\mathrm{X}$ \\
\hline VS6 & na & 57 & NA & $\mathrm{F}$ & $X$ & $X$ \\
\hline VS7 & na & 62 & 6.46 & $\mathrm{~F}$ & $X$ & $X$ \\
\hline VS8 & na & 54 & 3.34 & $\mathrm{~F}$ & $X$ & $X$ \\
\hline VS9 & na & 75 & 17.71 & $\mathrm{~F}$ & $\mathrm{X}$ & $\mathrm{X}$ \\
\hline VS10 & 699 & 50 & NA & $\mathrm{F}$ & $\mathrm{X}$ & $X$ \\
\hline VS11 & 1028 & 61 & NA & M & $\mathrm{X}$ & $X$ \\
\hline VS12 & 3478 & 58 & NA & M & $X$ & $X$ \\
\hline VS13 & 1084 & 66 & NA & M & $X$ & $X$ \\
\hline VS14 & 2170 & 28 & 1.20 & $\mathrm{~F}$ & $X$ & $X$ \\
\hline VS15 & 1079 & 64 & NA & M & $X$ & $X$ \\
\hline VS16 & 574 & 66 & 11.46 & M & $\mathrm{X}$ & $X$ \\
\hline VS17 & 560 & 53 & 1.46 & $\mathrm{~F}$ & $X$ & $X$ \\
\hline VS18 & 2371 & 69 & 1.67 & $\mathrm{~F}$ & $X$ & $X$ \\
\hline VS19 & 1499 & 61 & 0.12 & $\mathrm{M}$ & $X$ & \\
\hline VS20 & 280 & 44 & 0.62 & $\mathrm{M}$ & $X$ & \\
\hline VS21 & 1476 & 66 & 3.50 & M & $X$ & \\
\hline VS22 & 2968 & 80 & 0.41 & $\mathrm{M}$ & $X$ & \\
\hline VS23 & 1987 & 60 & 0.47 & M & $X$ & \\
\hline VS24 & 720 & 72 & 1.03 & $\mathrm{~F}$ & $X$ & \\
\hline VS25 & 811 & 68 & 0.14 & M & $X$ & \\
\hline VS26 & 1646 & 61 & 4.56 & $\mathrm{~F}$ & $X$ & \\
\hline VS27 & 1288 & 61 & 2.52 & M & $X$ & \\
\hline VS29 & na & 64 & 11.39 & $\mathrm{~F}$ & & $X$ \\
\hline VS30 & na & 39 & 11.99 & $\mathrm{~F}$ & & $X$ \\
\hline VS31 & na & 40 & 8.77 & $\mathrm{~F}$ & & $X$ \\
\hline VS33 & na & 59 & 7.11 & M & & $X$ \\
\hline VS34 & na & 33 & 6.89 & M & & $X$ \\
\hline VS35 & na & 30 & 15.05 & M & & $X$ \\
\hline VS36 & na & 45 & 5.41 & $\mathrm{~F}$ & & $X$ \\
\hline VS37 & na & 48 & 4.29 & $\mathrm{M}$ & & $X$ \\
\hline VS38 & na & 18 & 16.61 & $\mathrm{~F}$ & & $X$ \\
\hline VS39 & na & 58 & 9.70 & $\mathrm{~F}$ & & $X$ \\
\hline VS40 & na & 42 & 9.39 & M & & $X$ \\
\hline VS41 & na & 25 & 12.18 & $\mathrm{~F}$ & & $X$ \\
\hline VS42 & na & 45 & 18.42 & $\mathrm{~F}$ & & $X$ \\
\hline VS43 & na & 36 & 18.01 & $\mathrm{~F}$ & & $X$ \\
\hline VS44 & na & 58 & 7.87 & $\mathrm{~F}$ & & $X$ \\
\hline VS45 & na & 60 & 26.77 & $\mathrm{~F}$ & & $X$ \\
\hline VS46 & na & 33 & 11.68 & M & & $X$ \\
\hline VS48 & na & 42 & 5.46 & $\mathrm{M}$ & & $X$ \\
\hline VS49 & na & 54 & 18.21 & M & & $X$ \\
\hline VS50 & na & 63 & 3.20 & M & & $X$ \\
\hline VS51 & na & 47 & NA & M & & $X$ \\
\hline VS52 & na & 55 & 6.80 & M & & $X$ \\
\hline VS53 & na & 66 & 8.57 & M & & $X$ \\
\hline VS54 & na & 37 & 12.94 & $\mathrm{~F}$ & & X \\
\hline
\end{tabular}


Table 1 (continued)

\begin{tabular}{|c|c|c|c|c|c|c|}
\hline ID & $\mathrm{GKRS}^{1}$ & Age & Volume $^{2}$ & Sex & Microarray $^{3}$ & WES $^{4}$ \\
\hline VS55 & na & 26 & 10.40 & $\mathrm{~F}$ & & $X$ \\
\hline VS56 & na & 57 & 10.66 & M & & $X$ \\
\hline VS57 & na & 63 & 6.73 & M & & $\mathrm{X}$ \\
\hline VS58 & na & 42 & 5.01 & $\mathrm{~F}$ & & $X$ \\
\hline
\end{tabular}

${ }^{1}$ Time in days between Gamma Knife Radiosurgery and microsurgery

${ }^{2}$ Tumor volume in $\mathrm{cm}^{3}$

${ }^{3}$ Samples with DNA microarray data marked with $\mathrm{X}$

${ }^{4}$ Samples with WES data marked with $\mathrm{X}$

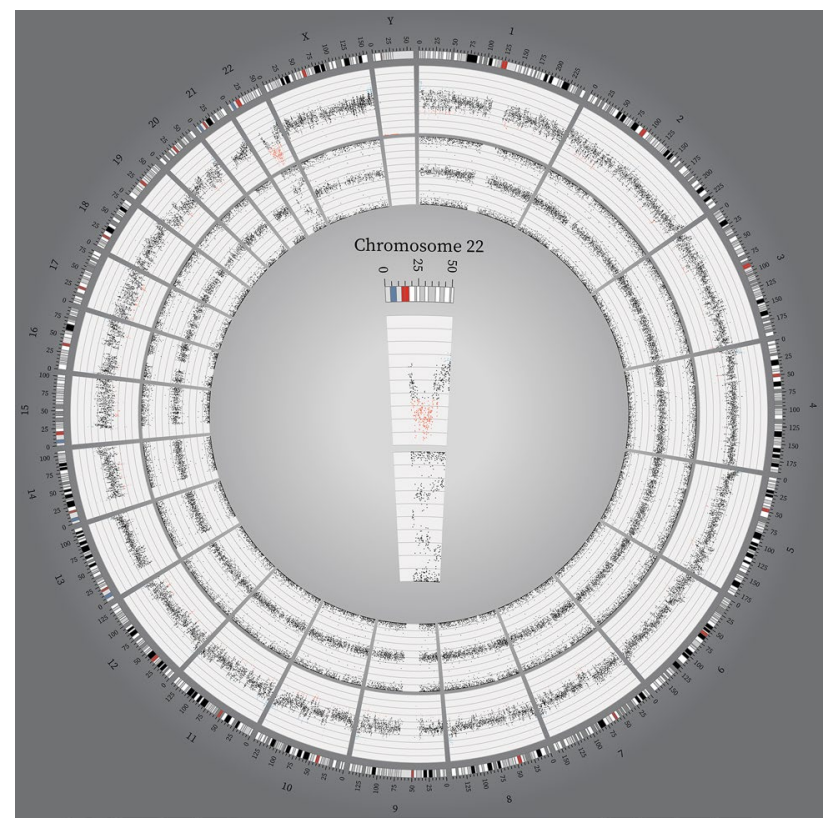

Fig. 1 Karyogram for sample VS10. Circos plot of copy number and single nucleotide polymorphism probe data for sample VS10, created using the Circos software [37]. The tracks from outside inwards: chromosome numbers, chromosomal position in $\mathrm{Mb}$, copy number and allele patterns. Copy number gains and losses are highlighted in blue and red, respectively. Most chromosomes show a continuous disomic copy number profile with a normal three band allele pattern (allele configurations $\mathrm{AA}, \mathrm{AB}$ and $\mathrm{BB}$ ). On chromosome 22, highlighted in the middle, we see an allelic loss (allele configuration A0 and $\mathrm{B} 0$ ) in the region of $N F 2$ followed by a CNN-ROH (allele configuration $\mathrm{AA}$ and $\mathrm{BB}$ ). However, the aberrations are only present in $63 \%$ of the cells giving rise to the split in the middle line of the allele pattern

the Nexus Copy Number built-in comparison analysis, we did not identify any CNA or gene associated with radiation exposure. We sought specifically for CNAs affecting genes coding for enzymes annotated to function in DNA repair pathways. Using the union call set, four tumors (two irradiated and two radiation-naïve) harbored CNAs affecting DNA repair genes. No difference between the groups was seen $\left(x^{2}=0.04, p=0.58\right)$. The results were similar for the ASCAT call set.
Table 2 Chromosome 22 aberrations

\begin{tabular}{|c|c|c|}
\hline ID & Aberration & $\begin{array}{l}\text { Aberrant } \\
\text { cell frac- } \\
\text { tion }\end{array}$ \\
\hline VS1 & $22 q 11.1 q 13.33(16052530-51244019) \times 1$ & 0.86 \\
\hline VS8 & $22 q 11.1 q 13.33(16052530-51244019) \times 1$ & 0.46 \\
\hline VS9 & 22q11.1q13.33(17922735-51244019) hmz & 0.46 \\
\hline VS10 & $\begin{array}{l}\text { 22q11.1q13.33(19639383-37988033) × } 1 \\
\text { 22q11.1q13.1(37988034-51244019) hmz }\end{array}$ & 0.63 \\
\hline VS13 & $22 \mathrm{q} 11.1 \mathrm{q} 13.33(16052530-51244019) \times 1$ & 0.61 \\
\hline VS16 & $22 q 11.1 q 13.33(16052530-51244019) \times 1$ & 0.46 \\
\hline VS17 & $22 \mathrm{q} 11.1 \mathrm{q} 13.33(16052530-51244019) \times 1$ & 0.29 \\
\hline VS20 & $22 q 11.1 q 13.33(16052530-51244019) \times 1$ & 0.26 \\
\hline VS22 & $22 \mathrm{q} 11.1 \mathrm{q} 13.33(16052530-51244019) \times 1$ & 0.45 \\
\hline VS27 & 22q11.21q13.33(18581773-51244019) hmz & 0.25 \\
\hline VS33 & $22 q 11.1 q 13.33(16157603-51220938) \times 1$ & 0.63 \\
\hline VS34 & $22 q 11.1 q 13.33(16157940-51237063) \times 1$ & 0.59 \\
\hline VS37 & $22 q 11.1 q 13.33(16157827-51220938) \mathrm{hmz}$ & 0.36 \\
\hline VS38 & $22 q 11.23 q 12.3(24167473-33156768) \mathrm{hmz}$ & NA \\
\hline VS42 & $22 q 11.1 q 13.33(16157762-51220938) \times 1$ & 0.27 \\
\hline VS43 & $22 q 11.1 q 13.33(20761063-51220938) \times 1$ & 0.94 \\
\hline VS45 & $22 q 11.1 q 13.33(16157623-51237063) \times 1$ & 0.79 \\
\hline VS46 & $22 q 11.22 q 13.33(22313733-51237063) \mathrm{hmz}$ & NA \\
\hline VS50 & $22 q 11.1 q 13.33(16157622-51237063) \times 1$ & 0.77 \\
\hline VS51 & $22 q 11.1 q 13.33(16157603-51219006) \times 1$ & 0.66 \\
\hline VS53 & $22 q 11.1 q 13.33(26688838-51237063) \times 1$ & 0.8 \\
\hline VS54 & $22 q 11.1 q 13.33(16157603-51237063) \times 1$ & NA \\
\hline VS55 & 22q11.1q13.33(16157771-51220938) hmz & NA \\
\hline VS57 & $22 q 11.23 q 13.33(23523234-51220938) \mathrm{hmz}$ & 0.4 \\
\hline VS58 & $22 q 11.1 q 13.33(16269779-51216564) \times 1$ & 0.84 \\
\hline
\end{tabular}

Chromosome 22 aberrations identified in VS. The naming of the aberrations starts with chromosome number followed by band, location in bp and type of aberration ( $\mathrm{x} 1$ for hemizygous loss and hmz for copy number neutral run of homozygosity). The last column gives the fraction of cells harboring the aberration 


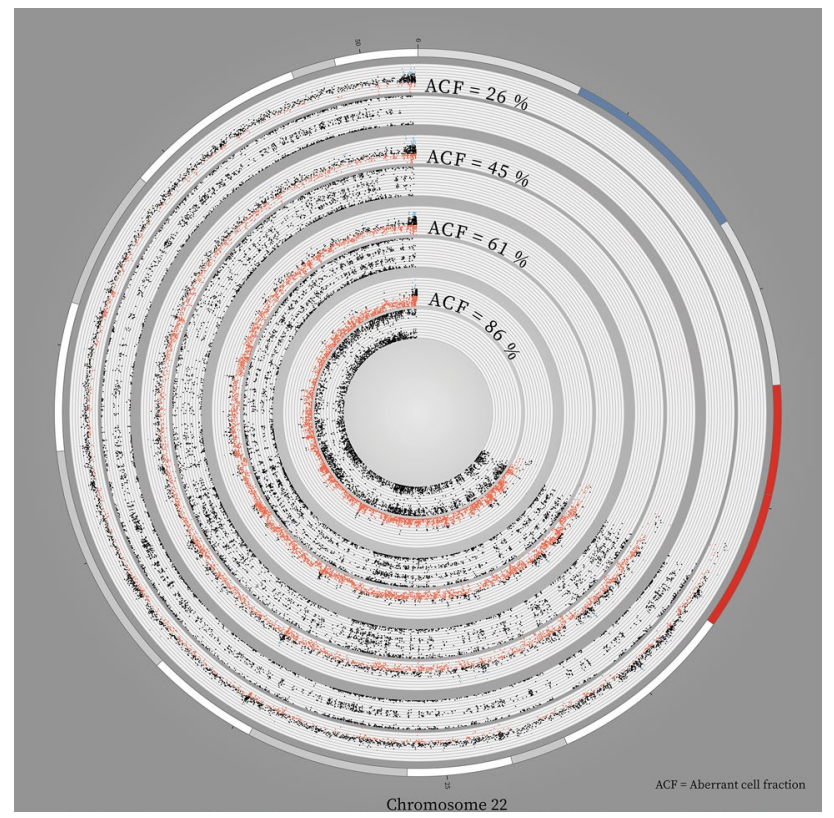

Fig. 2 Vestibular schwannoma consist of more than one major genetic clone. Circos plot of copy number and single nucleotide polymorphism probes in chromosome 22 for four vestibular schwannomas with increasing aberrant cell fraction. The tracks from outside inwards: chromosomal position in $\mathrm{Mb}$ on chromosome 22, copy number and allele patterns respectively for four vestibular schwannomas with increasing aberrant cell fraction. All samples demonstrate hemizygous loss of chromosome 22. The outermost sample shows a minor drop in copy number and a barely visible split in the middle line in the allele pattern because only $26 \%$ of the cells are aberrated. Moving inwards, the copy number drops and the split in the allele pattern increases, demonstrating an increase in aberrant cell fraction

\section{Discussion}

GKRS has become increasingly popular in treating VS over the past decades [21]. Several case reports have questioned its safety regarding malignant degeneration of benign tumors and inducing new neoplasms. For a review, see [9]. A dominating theory explaining the relationship between ionizing radiation dose and harmful effects is called the "linear no-threshold model" [22]. Although disputed, this theory explains that there is no safe limit, and that even a small amount of radiation might damage the DNA and initiate tumorigenesis. Standard treatment protocol with GKRS delivers 12 Gy to the periphery with a sharp decrease in the amount of energy delivered to the surrounding tissue. In the focus of the radiation, the dosage will be enough to initiate necrosis. In the periphery, we hypothesize that the dosage will harm the DNA and lead to one of the following: (1) detrimental DNA damage leading to apoptosis, (2) DNA mutations with tumorigenic potential, (3) DNA mutations with other or no effect at all. Although it seems theoretically feasible for GKRS to increase the risk of malignancy, epidemiologic studies do not support this [10, 23].

Previous studies have reported different genetic findings associated with radiation in VS. Lee et al. analyzed $30 \mathrm{sVS}$, including 4 irradiated tumors, utilizing microsatellite analysis to find that chromosome 22 aberration was more common in the radiation-naïve tumors [24]. Warren et al. found, using comparative genomic hybridization, that among 10 neurofibromatosis type 2 patients, radiation was associated with chromosomal aberrations [25]. In a recent study, Aaron et al. used WES on 12 VS, including two irradiated, to conclude that irradiated VS have increased copy number events and mutational burden [26]. One irradiated tumor harbored 184 mutations whereas the average across the cohort was 18.5. However, in our previous study utilizing WES on 46 VS, including 8 irradiated tumors, we also demonstrated one outlier, but this was radiation-naïve [7]. Taken together, this weakens the association between radiation exposure and hypermutated tumors.

This is the largest study investigating the effect of GKRS on the sVS genome. Using hierarchical clustering of the
Fig. 3 GKRS treatment does not affect the genomic CNA profile of vestibular schwannoma. Dendrogram of hierarchical clustering of the autosomal CNA patterns of vestibular schwannomas. Irradiated and radiation-naïve tumors depicted as red and black terminal vertical lines respectively. The clusters are not associated with previous radiation exposure

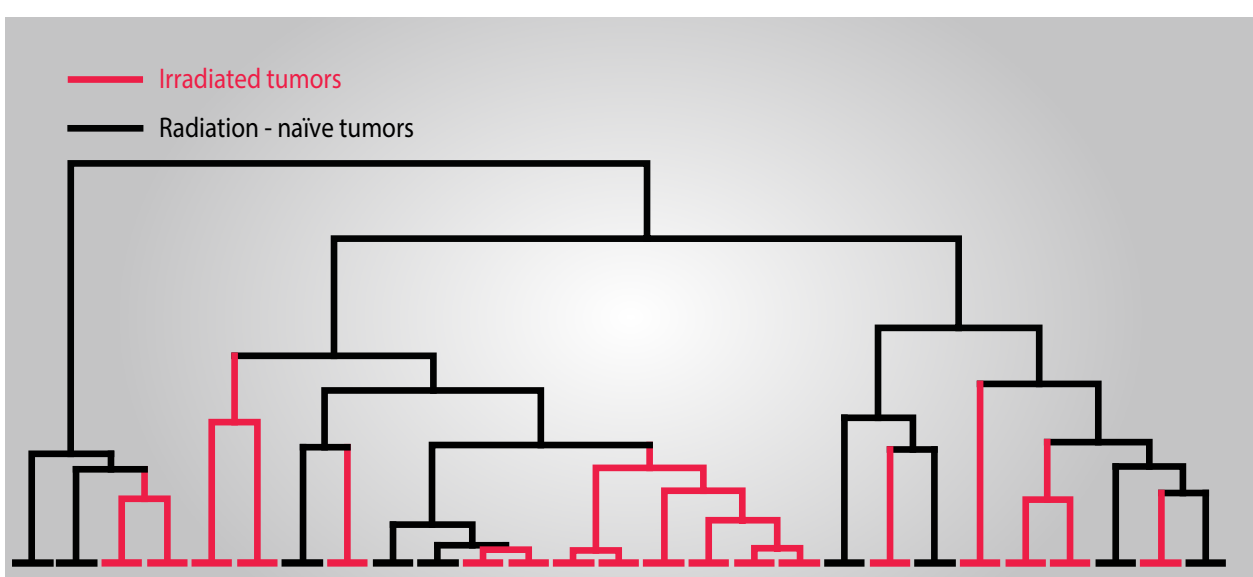


genome-wide CNA profiles, we did not identify any clustering based on radiation exposure. Neither did we find any gene or genomic loci that correlated with radiation exposure. The discrepancy with previous studies on the subject might be due to larger sample size and the method used. In a recent paper, 18 radiation-induced meningiomas were analyzed for tumor-specific CNAs [27]. A mean total of $22 \%$ of the exome was affected by CNA. This is in stark contrast with our irradiated tumors exhibiting a median of $0.14 \%$ of the autosome covered by CNA. The meningioma patients had received cranial radiotherapy for diseases like medulloblastoma and central nervous system lymphoma, a therapy that delivers higher radiation doses to healthy tissue compared to GKRS. This comparison lends support to the fact that GKRS does not induce collateral damage to the extent seen after conventional radiotherapy. We expect that GKRS causes mutations in the normal tissue surrounding VS. However, the mutations induced need to provide a selective growth advantage to the affected cell initiating a clonal expansion for it to be detected using bulk DNA analysis and even for it to be clinically relevant. It is feasible that a growth advantage might be obtained, but that it is very rare in agreement with epidemiologic studies and our study.

Between 5 and $10 \%$ of sVSs do not respond to GKRS treatment. The GKRS response might depend on both treatment and tumor factors. Studying the tumor factors might elucidate the mechanism of radioresistance as well as identify biomarkers. Archibald et al. found a higher expression of the immune-related protein B7-H1 among irradiated sVS, but no difference at RNA level [28]. This might be a consequence of the radiation induced inflammation and hence not connected to the cause of the radioresistance. Through the use of genome-wide association studies, gene expression and DNA sequencing, several biomarkers of radiotherapy treatment response have been found in neoplasms [29-32]. It has been postulated that enhanced DNA repair mechanisms lead to radiotherapy treatment failure. Hence, we sought to evaluate whether the radioresistant VSs harbored CNAs in DNA repair genes. Although we identified some impaired DNA repair genes, they were distributed equally among the irradiated and radiation-naïve tumors. We did not find any other gene or genomic loci associated with radioresistance. One pitfall of our study is that we do not have positive controls for tumors that respond to GKRS treatment as these are not surgically removed. However, as GKRS treatment is effective in 90-95\% of sVSs, we believe that the radiationnaïve tumors included in this study serve as a viable surrogate for GKRS treatment responders. Also, to detect any genetic aberrations caused by ionizing radiation, a longitudinal study design using paired samples of radiation-naïve and irradiated tumors would be the most sensitive. However, we believe our study design would be able to detect any large effects ionizing radiation.
Among the chromosome 22 aberrated tumors, a median of $59 \%$ of the cells harbored the CNA, suggesting that VS consists of more than one major clone. This is in accordance with our previous study on small mutations, where we reported a median NF2 variant allele frequency of $24 \%$ [7]. Considering the bias of ASCAT only calling heterogeneity in tumors with large aberration and the variant allele frequencies reported in our WES study, it seems likely that most or all VSs consists of more than one major genetic clone. A recent study by Lewis et al. found that tumor-associated macrophages constituted $50-60 \%$ of the cells in eight growing VSs [33]. Hence, infiltrating macrophages might constitute the clone coexisting with the neoplastic cells. Further on, Lewis et al. found that macrophages accounted for the proliferating cells in VS. However, we did not find any association between aberrant cell fraction, which might correlate inversely with the macrophage fraction, and tumor growth. The finding of intratumor genetic heterogeneity has significant implications for molecular studies on VS. We saw from our data that the lower the aberrant cell fraction, the higher the number of CNAs called. This implies a problem with the software and theoretic framework underlying calling of aberrations. The possibly large fraction of infiltrating macrophages would also preclude other molecular studies using bulk analyzing, like transcriptome and proteome studies. A possible way to bypass this could be to apply singlecell analysis to provide a better understanding VS molecular biology.

Carlson et al. recently profiled structural variants in sVS using whole-genome sequencing to find biallelic inactivation of the NF2 gene in all 22 sVSs analyzed [8]. Previous studies have demonstrated NF2 variants in $15-84 \%$ of the analyzed tumors [34]. Combining our data from whole-genome microarray, WES and MLPA, we found at least one variant in NF2 in $83 \%$ of the tumors. This discrepancy might reflect differences in the detection limit of the methods used or variations in the study population. Carlson et al. also found that VSs with severe phenotype tended to harbor large structural variants outside chromosome 22. However, we did not find any association with specific CNAs and previous radiation exposure, tumor size or age. In agreement with Carlson et al. we did not find any recurrent focal alterations and it seems unlikely that this kind of genetic event plays a significant role in VS tumorigenesis [8]. Previous studies have identified recurrent non-chromosome 22 regions affected by CNA, like $9 q 34,17 q, 19,16 q$ and $9 p 21[25,35]$. We found a total of 38 genomic loci affected in three or more tumors. However, all but chromosome 22 aberration were normal variants present in healthy subjects.

In our previous study, we analyzed a total of $46 \mathrm{VS}$, including 8 irradiated VS, with WES and MLPA to conclude that radiation exposure or radiosensitivity is not associated with increased mutational burden or specific small 
mutations [7]. Ionizing radiation is known to induce DNA double-strand breakage resulting in CNA [36]. To address this issue, we analyzed an extended set of irradiated samples with whole-genome DNA microarray to conclude that neither specific CNAs, nor the genomic CNA profile play a role either. The methods used in these studies are not capable of detecting structural variants not affecting gene dosage or heterozygosity (e.g. inversions and translocations). Hence, future studies should address this as well as epigenetic mechanisms to elucidate the molecular consequences of ionizing radiation in VS as well as markers of radioresistance.

\section{Conclusions}

We did not find any CNA or genomic CNA profile associated with radiation exposure in VS. This finding lends support to the low risk of GKRS. We demonstrated that VS exhibit intratumor heterogeneity and further studies are warranted to elucidate whether it is different tumor clones or normal cell infiltration. In our study, the only recurrent CNA in VS is hemizygous loss or copy number neutral loss of heterozygosity on chromosome 22.

Acknowledgements We thank Ms. Guri Matre and Mr. Atle Brendehaug at Center for Medical Genetics and Molecular Medicine for technical assistance and Ms. Monica Katrine Finnkirk at the National Center for Vestibular Schwannoma Treatment and the Department of Neurosurgery for administrative work

\section{Funding Open Access funding provided by University of Bergen}

Open Access This article is licensed under a Creative Commons Attribution 4.0 International License, which permits use, sharing, adaptation, distribution and reproduction in any medium or format, as long as you give appropriate credit to the original author(s) and the source, provide a link to the Creative Commons licence, and indicate if changes were made. The images or other third party material in this article are included in the article's Creative Commons licence, unless indicated otherwise in a credit line to the material. If material is not included in the article's Creative Commons licence and your intended use is not permitted by statutory regulation or exceeds the permitted use, you will need to obtain permission directly from the copyright holder. To view a copy of this licence, visit http://creativecommons.org/licenses/by/4.0/.

\section{References}

1. Stepanidis K, Kessel M, Caye-Thomasen P, Stangerup SE (2014) Socio-demographic distribution of vestibular schwannomas in Denmark. Acta Otolaryngol 134(6):551-556

2. Evans DG, Moran A, King A, Saeed S, Gurusinghe N, Ramsden $\mathrm{R}$ (2005) Incidence of vestibular schwannoma and neurofibromatosis 2 in the North West of England over a 10-year period: higher incidence than previously thought. Otol Neurotol 26(1):93-7

3. Carlson ML, Tveiten OV, Driscoll CL, Goplen FK, Neff BA, Pollock BE et al (2015) What drives quality of life in patients with sporadic vestibular schwannoma? Laryngoscope 125(7):1697-1702

4. Sainz J, Huynh DP, Figueroa K, Ragge NK, Baser ME, Pulst SM (1994) Mutations of the neurofibromatosis type 2 gene and lack of the gene product in vestibular schwannomas. Hum Mol Genet 3(6):885-891

5. Trofatter JA, MacCollin MM, Rutter JL, Murrell JR, Duyao MP, Parry DM et al (1993) A novel moesin-, ezrin-, radixin-like gene is a candidate for the neurofibromatosis 2 tumor suppressor. Cell 72(5):791-800

6. Agnihotri S, Jalali S, Wilson MR, Danesh A, Li M, Klironomos $\mathrm{G}$ et al (2006) The genomic landscape of schwannoma. Nat Genet 48:1339-1348

7. Havik AL, Bruland O, Myrseth E, Miletic H, Aarhus M, Knappskog PM et al (2017) Genetic landscape of sporadic vestibular schwannoma. J Neurosurg 128:911-922

8. Carlson ML, Smadbeck JB, Link MJ, Klee EW, Vasmatzis G, Schimmenti LA (2018) Next generation sequencing of sporadic vestibular schwannoma: necessity of biallelic NF2 inactivation and implications of accessory Non-NF2 variants. Otol Neurotol 39:e860-e871

9. Patel TR, Chiang VL (2014) Secondary neoplasms after stereotactic radiosurgery. World Neurosurg 81(3-4):594-599

10. Carlson ML, Glasgow AE, Jacob JT, Habermann EB, Link MJ (2016) The short-term and intermediate-term risk of second neoplasms after diagnosis and treatment of unilateral vestibular schwannoma: analysis of 9460 cases. Int J Radiat Oncol Biol Phys 95(4):1149-1157

11. Travis LB, Ng AK, Allan JM, Pui CH, Kennedy AR, Xu XG et al (2014) Second malignant neoplasms and cardiovascular disease following radiotherapy. Health Phys 106(2):229-246

12. Sass H, Caye-Thomasen P (2018) Contemporary molecular biology of sporadic vestibular schwannomas: a systematic review and clinical implications. J Int Adv Otol 14:322

13. Mayrhofer M, Viklund B, Isaksson A (2016) Rawcopy: Improved copy number analysis with Affymetrix arrays. Sci Rep 6:36158

14. Thorvaldsdottir H, Robinson JT, Mesirov JP (2013) Integrative genomics viewer (IGV): high-performance genomics data visualization and exploration. Brief Bioinform 14(2):178-192

15. Quinlan AR, Hall IM (2010) BEDTools: a flexible suite of utilities for comparing genomic features. Bioinformatics 26(6):841-842

16. MacDonald JR, Ziman R, Yuen RK, Feuk L, Scherer SW (2014) The database of genomic variants: a curated collection of structural variation in the human genome. Nucleic Acids Res 42(Database issue):D986-D992

17. Mermel CH, Schumacher SE, Hill B, Meyerson ML, Beroukhim R, Getz G (2011) GISTIC2.0 facilitates sensitive and confident localization of the targets of focal somatic copy-number alteration in human cancers. Genome Biol 12(4):R41

18. Van Loo P, Nordgard SH, Lingjaerde OC, Russnes HG, Rye IH, Sun W et al (2010) Allele-specific copy number analysis of tumors. Proc Natl Acad Sci USA 107(39):16910-16915

19. Favero F, Joshi T, Marquard AM, Birkbak NJ, Krzystanek M, Li Q et al (2015) Sequenza: allele-specific copy number and mutation profiles from tumor sequencing data. Ann Oncol 26(1):64-70

20. R Core Team (2018) R: a language and environment for statistical computing. R Foundation for Statistical Computing, Vienna

21. Carlson ML, Habermann EB, Wagie AE, Driscoll CL, Van Gompel JJ, Jacob JT et al (2015) The changing landscape of vestibular schwannoma management in the United Statesa shift toward conservatism. Otolaryngol Head Neck Surg 153(3):440-446

22. Shah DJ, Sachs RK, Wilson DJ (2012) Radiation-induced cancer: a modern view. Br J Radiol 85(1020):e1166-e1173

23. Pollock BE, Link MJ, Stafford SL, Parney IF, Garces YI, Foote RL (2017) The risk of radiation-induced tumors or malignant 
transformation after single-fraction intracranial radiosurgery: results based on a 25-year experience. Int J Radiat Oncol Biol Phys 97(5):919-923

24. Lee DJ, Maseyesva B, Westra W, Long D, Niparko JK, Califano J (2006) Microsatellite analysis of recurrent vestibular schwannoma (acoustic neuroma) following stereotactic radiosurgery. Otol Neurotol 27(2):213-9

25. Warren C, James LA, Ramsden RT, Wallace A, Baser ME, Varley $\mathrm{JM}$ et al (2003) Identification of recurrent regions of chromosome loss and gain in vestibular schwannomas using comparative genomic hybridisation. J Med Genet 40(11):802-806

26. Aaron KA, Manojlovic Z, Tu N, Xu Y, Jin Y, Chang S et al (2020) What genes can tell: a closer look at vestibular schwannoma. Otol Neurotol 41(4):522-9

27. Agnihotri S, Suppiah S, Tonge PD, Jalali S, Danesh A, Bruce JP et al (2017) Therapeutic radiation for childhood cancer drives structural aberrations of NF2 in meningiomas. Nat Commun $8(1): 186$

28. Archibald DJ, Neff BA, Voss SG, Splinter PL, Driscoll CL, Link MJ et al (2010) B7-H1 expression in vestibular schwannomas. Otol Neurotol 31(6):991-7

29. Ma J, Setton J, Morris L, Albornoz PB, Barker C, Lok BH et al (2017) Genomic analysis of exceptional responders to radiotherapy reveals somatic mutations in ATM. Oncotarget 8(6):10312-10323

30. Scott JG, Berglund A, Schell MJ, Mihaylov I, Fulp WJ, Yue B et al (2017) A genome-based model for adjusting radiotherapy dose (GARD): a retrospective, cohort-based study. Lancet Oncol 18(2):202-211

31. Skinner HD, Sandulache VC, Ow TJ, Meyn RE, Yordy JS, Beadle BM et al (2012) TP53 disruptive mutations lead to head and neck cancer treatment failure through inhibition of radiation-induced senescence. Clin Cancer Res 18(1):290-300

32. West CM, Barnett GC (2011) Genetics and genomics of radiotherapy toxicity: towards prediction. Genome Med 3(8):52

33. Lewis D, Roncaroli F, Agushi E, Mosses D, Williams R, Li KL et al (2018) Inflammation and vascular permeability correlate With growth in sporadic vestibular schwannoma. Neuro-oncology 21:314-325

34. de Vries M, van der Mey AG, Hogendoorn PC (2015) Tumor biology of vestibular schwannoma: a review of experimental data on the determinants of tumor genesis and growth characteristics. Otol Neurotol 36(7):1128-36

35. Koutsimpelas D, Felmeden U, Mann WJ, Brieger J (2011) Analysis of cytogenetic aberrations in sporadic vestibular schwannoma by comparative genomic hybridization. J Neuro-oncol 103(3):437-443

36. Sholl LM, Barletta JA, Hornick JL (2017) Radiation-associated neoplasia: clinical, pathological and genomic correlates. Histopathology 70(1):70-80

37. Krzywinski M, Schein J, Birol I, Connors J, Gascoyne R, Horsman $\mathrm{D}$ et al (2009) Circos: an information aesthetic for comparative genomics. Genome Res 19(9):1639-1645

Publisher's Note Springer Nature remains neutral with regard to jurisdictional claims in published maps and institutional affiliations. 